\title{
A Quantum Circuit for Three Quantum Bits to Produce Quantum Entanglement States (ML- Quadripartite)
}

\author{
Mezher B. Saleh $^{* 1}$ and Loay E. George ${ }^{* * 2}$ \\ *Department of Physics, College of Science, Al- Mustansiriyah University. \\ ** Department of Computer Science, College of Science, Baghdad University. \\ 1E-mail: mezherbaker@yahoo.com. \\ ${ }^{2}$ E-mail: loayedwar57@ @cbaghdad.edu.iq.
}

\begin{abstract}
In this research, a quantum system consists of three qubits is considered; it has eight states $(|000>| 001>,,|010>| 011>,,|100>| 101>,, \mid 110>$ and $\mid 111>)$. Each of these states is passed through a quantum circuit made of a sequence of quantum gates. The circuit applies $\mathrm{X}$ - gate on the first qubit from the left, then using CNOT-gate on the first and second qubits and using the Hadamard-gate on the first qubit. Finally, Hadamard-gate is applied on the third qubit. When the state $1000>$ is passed as input through the proposed quantum circuit then the output includes four entanglement states; for the other input states the output will be another non-repeatable entanglement states. So, for using all states in quantum circuit the result will be eight equations each one consists of four entanglement states; which called ML- Quadripartite.
\end{abstract}

Keywords: Quantum Bit, X - Gate, Hadamard - Gate, CNOT- Gate, Quantum Circuit.

\section{Introduction}

The aim of this research to study the effect of a proposed quantum system consists of quantum gates on a quantum system consist of 3 qubits to produce a set of entanglement states. In quantum mechanics the entanglement is very important. In the literature, the entanglement states for two qubit have been introduced by applying a quantum circuit include Hadamard-gate and CNOT-gate; this introduced circuit had generated the Bell states (or EPR pairs) [1, 2].

In 1998, Plenio and Vedral have discussed a special aspect of quantum mechanics, namely the possibility of entanglement between two or more particles. They established the basic properties of entanglement using quantum state teleportation. These principles allowed them to formulate quantitative measures of entanglement. In this paper we will show that the same general principles can also be used to easily investigate the seemingly difficult questions regarding entanglement dynamics. This may be useful to manipulate thermodynamics of entanglement [3].

In 2004, Macchiavello gave a tutorial review on the role of entanglement of quantum systems in some tasks of quantum information theory and described in particular how it can be exploited to achieve secure communication channels [4].

In 2009 Felloni and Strini have explored the entanglement in composite quantum systems, and how its peculiar properties are exploited in quantum information and communication protocols by means of Diagrams of States; which is, a novel method to graphically represent and analyze how quantum information is elaborated during computations performed by quantum circuits. They presented quantum diagrams of states for Bell states generation, measurements and projections, for dense coding and quantum teleportation, for probabilistic quantum machines designed to perform approximate quantum cloning and universal NOT and, finally, for quantum privacy amplification based on entanglement purification [5].

\section{Theoretical Basis}

Quantum entanglement is an attractive and key idea from the quantum field that has no counterpart in the classical world. The qubit $|\psi\rangle$ is said to be entangled if it cannot be written in the product state $[2,6,7]$. The entanglement quantum system can be obtained using quantum circuits. The quantum circuit can be modeled using from the quantum gates; in this study the X-gate, Hadamard-gate and CNOT-gate have been used. 
The Pauli-X Gate or quantum NOT gate for qubits can be defined as a process that take $\mid 0>$ and produces $\mid 1>$ and vice-versa. However in case of superposition, the NOT gate acts linearly on the state $\alpha|0>+\beta| 1>$ to produce the state $\alpha|1>+\beta| 0>$ [8].

The matrix representation of quantum NOT gate is:

$X=\sigma_{x}=\left[\begin{array}{ll}0 & 1 \\ 1 & 0\end{array}\right]$

And the matrix representation of the state

$\alpha|0>+\beta| 1>i s\left[\begin{array}{l}\alpha \\ \beta\end{array}\right]$.

If the quantum NOT gate is applied on the state $\alpha|0>+\beta| 1>$ then:

$X\left[\begin{array}{l}\alpha \\ \beta\end{array}\right]=\left[\begin{array}{ll}0 & 1 \\ 1 & 0\end{array}\right]\left[\begin{array}{l}\alpha \\ \beta\end{array}\right]=\left[\begin{array}{l}\beta \\ \alpha\end{array}\right]=$

So that Pauli $-\mathrm{X}$ Gate $=$ Quantum NOT Gate $=$ bit flip

The other gate is Hadamard gate acts on a single qubit. The matrix representation of Hadamard gate is defined as [9]:

$H=\frac{1}{\sqrt{2}}\left[\begin{array}{cc}1 & 1 \\ 1 & -1\end{array}\right]$

For the $\mid 0>$ output is $\frac{1}{\sqrt{2}}(|0>+| 1>)$ and for $\mid 1>$ the output is $\frac{1}{\sqrt{2}}(|0>-| 1>)$. Here, the CNOT- Gate is also used. The CNOT-gate is the prototype of multi qubit quantum gate. The CNOT Gate has two input qubits:

i. Control Qubit: the control bit is represented by first qubit from the left.

ii. Target Qubit: The target bit is represented by second qubit.

The CNOT gate works in the way: "if the control qubit is set to 0 then the target qubit remains unaltered. If the control qubit is set to 1 , then the target qubit is inverted. This will change the input state into a set of states [9].

\section{Entanglement States for Three Qubits System}

A system consists of three qubits has eight quantum states (i.e., the number of states $=2^{3}=8$; these states are $1000>, \mid 001>$, $|010>| 011>,,|100>| 101>,, \mid 110>$ and |111>.
To get the entanglement state the quantum gates must be applied but with a certain order.

Case 1 (for input state $\mid 000>$ ): the quantum circuit to produce the entanglement state is shown in Fig.(1).

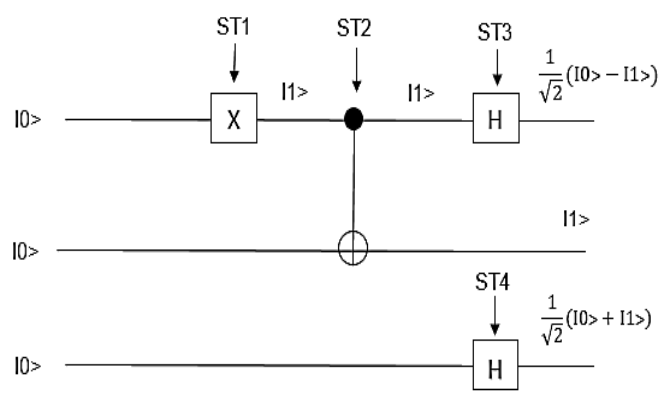

\section{Fig.(1): The entanglement state output for the input state $\mid 000>$.}

Several stages are applied to get the entanglement state from state $1000>$ (as shown in Fig.(1)). These stages are:

Stage (1): in this stage apply X-gate on qubit 0:

$\mathrm{X}|0>\rightarrow| 1>$

Stage (2): in this stage the CNOT-Gate is applied on state $\mid 10>$ :

$$
\begin{aligned}
& \text { CNOT - Gate } \mid 10>=\left[\begin{array}{llll}
1 & 0 & 0 & 0 \\
0 & 1 & 0 & 0 \\
0 & 0 & 0 & 1 \\
0 & 0 & 1 & 0
\end{array}\right]\left[\begin{array}{l}
0 \\
0 \\
1 \\
0
\end{array}\right] \\
& =\left[\begin{array}{l}
0 \\
0 \\
0 \\
1
\end{array}\right]=\mid 11>
\end{aligned}
$$

Stage (3): the third stage is applying Hadamard gate on qubit $\mid 1>$ :

$$
\begin{aligned}
H-\text { Gate } \mid 1> & =\frac{1}{\sqrt{2}}\left[\begin{array}{cc}
1 & 1 \\
1 & -1
\end{array}\right]\left[\begin{array}{l}
0 \\
1
\end{array}\right] \\
& =\frac{1}{\sqrt{2}}(|0>-| 1>)
\end{aligned}
$$

Stage (4): in this stage the $\mathrm{H}$-Gate is applied on qubit $\mid 0>$ :

$$
\begin{aligned}
H-\text { Gate } \mid 0> & =\frac{1}{\sqrt{2}}\left[\begin{array}{cc}
1 & 1 \\
1 & -1
\end{array}\right]\left[\begin{array}{l}
1 \\
0
\end{array}\right] \\
& =\frac{1}{\sqrt{2}}(|0>+| 1>)
\end{aligned}
$$

Stage (5): in this stage tensor product result of stage (3) with qubit $\mid 1>$ is applied:

$$
\frac{1}{\sqrt{2}}(|0>-| 1>) \otimes \mid 1>=\frac{1}{\sqrt{2}}(|01>-| 11>)
$$


Stage (6): In this is a final stage the tensor product is bone between the results of stage (5) and stage (4). The result of this stage represents the entanglement states. So that after all these stages we get:

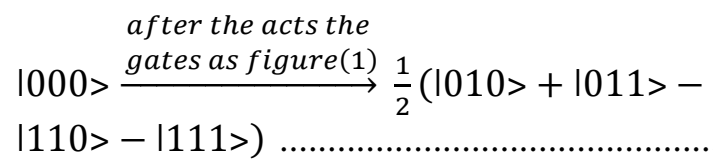

\section{Results and Discussion}

The above six stages have been programmed using Mathlab programming tool, as depicted in the following:

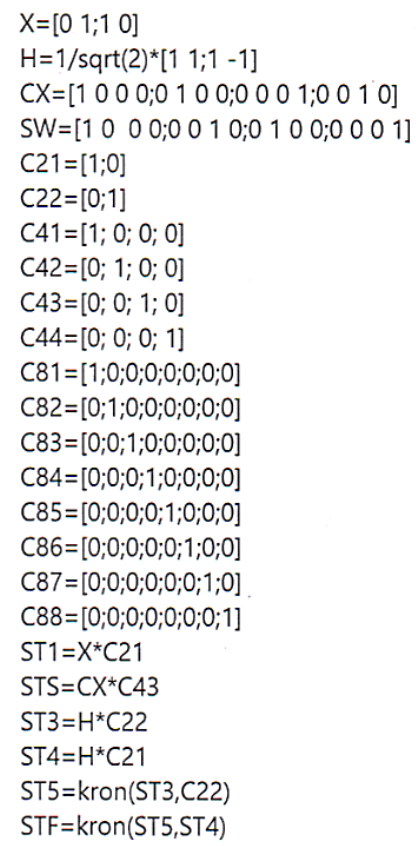

Case 2 (when the input state is $1001>$ ): same stages as in the state $1000>$ are applied to other states, so the output entanglement for input state $\mid 001>$ will be:

$\mid 001>\rightarrow \frac{1}{2}(|010>-| 011>-|110>+| 111>)$

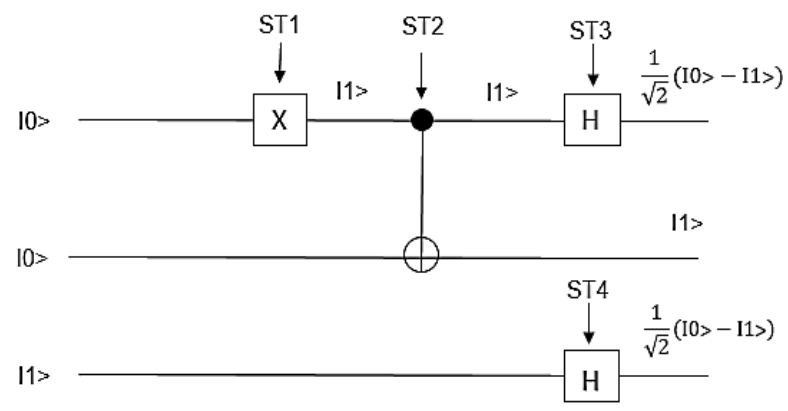

Fig.(2): The quantum circuit output for the state $\mid 001>$.
Case 3 (for state $\mid 010>$ ): As depicted in Fig.(3), the output entanglement state when this state is passed through the introduced quantum circuit is:

$$
\mid 010>\rightarrow \frac{1}{2}(|000>+| 001>-|100>-| 101>)
$$

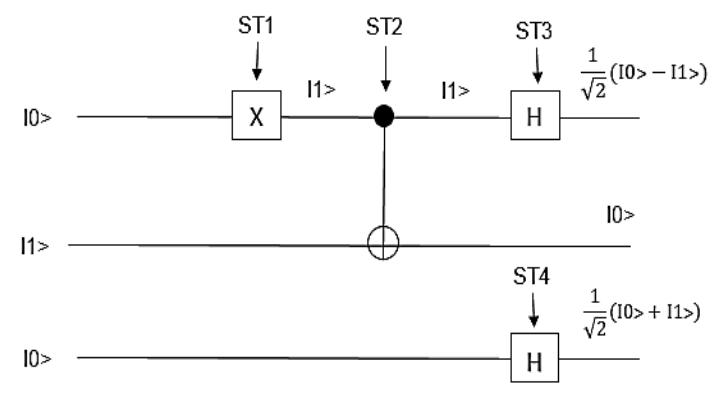

Fig.(3): The output of quantum circuit for the state $\mid 010>$.

Case 4 (for state |011>): as shown in Fig.(4), the entanglement states after passing the state |011> through the quantum circuit is:

$$
\mid 011>\rightarrow \frac{1}{2}(|000>-| 001>-|100>+| 101>)
$$

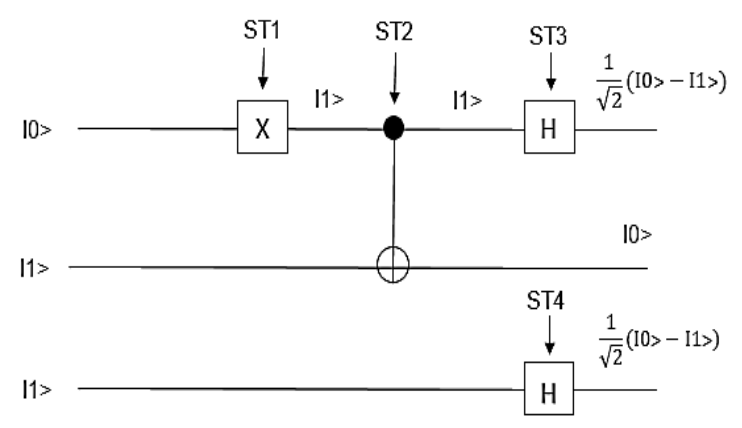

Fig.(4): The quantum circuit output for the state |011>.

Case 5 (for state $\mid 100>$ ): the entanglement state when this state passes through the quantum gates of the circuit is:

$$
\mid 100>\rightarrow \frac{1}{2}(|000>+| 001>+|100>+| 101>)
$$

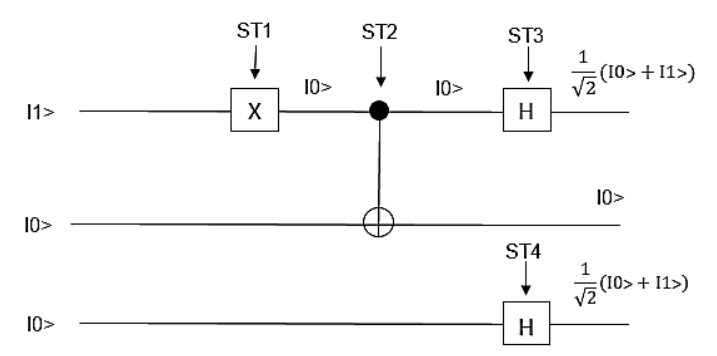

Fig.(5): The output of quantum circuit for the state $|100\rangle$. 
Case 6 (for state 1101): the output entanglement state for this input state is:

$$
\mid 101>\rightarrow \frac{1}{2}(|000>-| 001>+|100>-| 101>)
$$

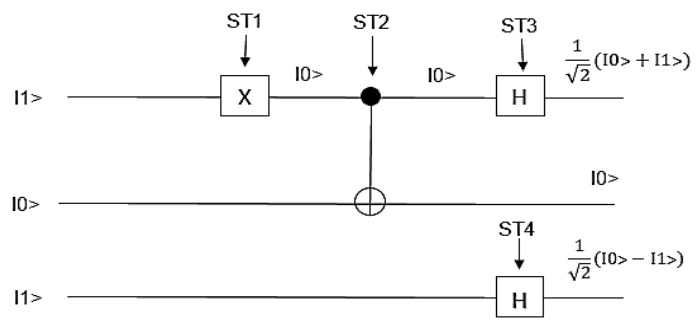

Fig.(6): Quantum circuit output for the state |101>.

Case 7 (for state $\mid 110>$ ): the output entanglement state in this case will be:

$$
\mid 110>\rightarrow \frac{1}{2}(|010>+| 011>+|110>+| 111>)
$$

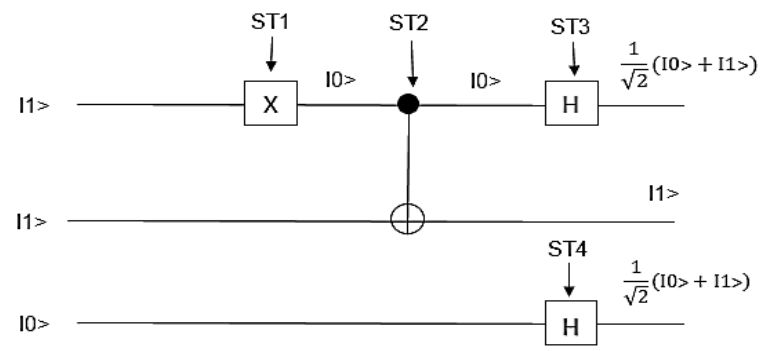

Fig.(7): Quantum circuit output for the state $\mid 110>$.

Table (1)

Input state and the corresponding output Entanglement states for three quits system.

\begin{tabular}{||c||c||}
\hline \hline Input States & Output Entanglement States \\
\hline \hline $\mid 000>$ & $\frac{1}{2}(|010>+| 011>-|110>-| 111>)$ \\
\hline \hline $\mid 001>$ & $\frac{1}{2}(|010>-| 011>-|110>+| 111>)$ \\
\hline \hline $\mid 010>$ & $\frac{1}{2}(|000>+| 001>-|100>-| 101>)$ \\
\hline $\mid 011>$ & $\frac{1}{2}(|000>-| 001>-|100>+| 101>)$ \\
\hline $\mid 100>$ & $\frac{1}{2}(|000>+| 001>+|100>+| 101>)$ \\
\hline \hline $\mid 101>$ & $\frac{1}{2}(|000>-| 001>+|100>-| 101>)$ \\
\hline \hline $\mid 110>$ & $\frac{1}{2}(|010>+| 011>+|110>+| 111>)$ \\
\hline \hline $\mid 111>$ & $\frac{1}{2}(|010>-| 011>+|110>-| 111>)$ \\
\hline \hline
\end{tabular}




\section{Conclusion}

When putting superposition state on the quantum circuit with certain arrangement of gates we get four states not product state so that those are entanglement states as in equation 9. When the input state different from another, a new case of tangles can produced as in equation $10,11,12131415$ and 16 . Through the use of the quantum circuit, and the introduction of eight states we can get to four states for each input state as shown in Table (1).

\section{References}

[1] Nielsen M., Chuang I., "Quantum Computation and Quantum Information", Canbridge University Press, Cambridge, England, 2010.

[2] Djordjevic I., "Quantum Information Processing and Quantum Error Correction", Elsevier, Great Britain, 2012.

[3] Plenio M., Vedral V., "Teleportation, Entanglement and Thermodynamics in the Quantum World", Contemporary Physics, 39 (6), 431-446, 1998.

[4] Macchiavello C., "On the Role of Entanglement in Quantum Information", Physica A, 338(1), 68-75, 2004.

[5] Felloni S., Strini G., "Entanglement based Quantum Computing by Diagrams of States", International Journal of Applied Mathematics and Computer Sciences, 5(4), 202-212, 2009.

[6] Marinescu D., Marinescu G., "Classical and Quantum Information”, Elsevier, 2012.

[7] Furusawa A., Loockn P., "Quantum Teleportation and Entanglement", Weinheim, Germany, 2011.

[8] Keyl M., "Fundamentals of Quantum Information Theory" Institute of Mathematical Physics, Braunschweig, Germany, 2008.

[9] Nayak S., Nayak Sh., Singh J., "An Introduction to Basic Logic Gates for Quantum Computer", International Journal of Advanced Research in Computer Science and Software Engineering, 3(10), 163-171, 2013.

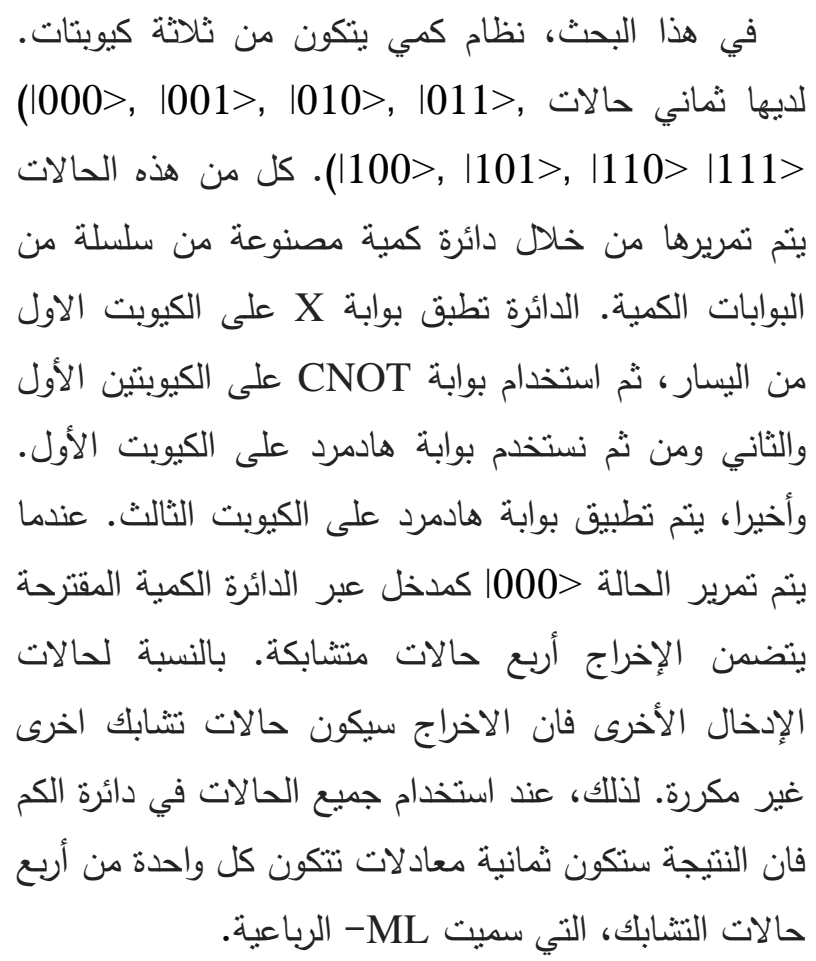

